\title{
Prothrombin time derived fibrinogen determination on Sysmex CA-6000 ${ }^{\mathrm{TM}}$
}

\author{
A S Lawrie, S J McDonald, G Purdy, I J Mackie, S J Machin
}

\begin{abstract}
Aim-To evaluate PT derived fibrinogen determinations with reference to the Clauss fibrinogen assay using a Sysmex CA-6000 ${ }^{\mathrm{TM}}$ random access coagulation analyser.

Methods-Samples were analysed from normal subjects $(n=20)$, patients with renal or liver dysfunction $(n=25)$, critically ill patients $(n=25)$, patients receiving oral anticoagulant treatment ( $n$ $=50$, and patients with a haemoglobinopathy $(n=127)$. Prothrombin times were performed using two thromboplastins: one derived from rabbit brain (Dade: Thromboplastin IS) and the other from recombinant human tissue factor (Dade: Innovin). Fibrinogen was assayed by the Clauss method using a commercial kit (Dade: Fibrinogen).
\end{abstract}

Results-The relation between Clauss fibrinogen and PT derived fibrinogen was found to be dependent on the patient's clinical group and source of the thromboplastin used. When the data from the above sample groups were pooled there was still a significant difference $(p<0.001)$ between Clauss fibrinogen and PT derived fibrinogen, irrespective of thromboplastin used.

Conclusions-It is unsafe to use the PT derived fibrinogen for patient monitoring owing to non-uniform variability in response to clinical status and reagent employed; however, it may prove to be a useful screening test in a research environment for estimating fibrinogen levels among defined patient groups.

(F Clin Pathol 1998;51:462-466)

Haemostasis Research Unit, Department of Haematology,

University College

London, London, UK

A S Lawrie

S J McDonald

G Purdy

I J Mackie

S J Machin

Correspondence to:

Dr A S Lawrie, Haemostasis Research Unit, Department of Haematology, University College London, 98 Chenies Mews, London WC1E 6HX, UK;

email: andrew.lawrie@ucl. ac.uk

Accepted for publication 12 March 1998
Keywords: prothrombin time; fibrinogen assay; haemostasis

Fibrinogen is an essential component of the haemostatic process, with key roles both in plasmatic clot formation ${ }^{1}$ and as a cofactor in the aggregation of platelets. $^{2}$ Severe fibrinogen deficiency can occur as an inherited disorder, ${ }^{3}$ as a result of reduced synthesis secondary to liver failure, ${ }^{4}$ and during consumptive coagulopathies, ${ }^{5}$ and may result in a life threatening haemorrhagic diathesis. ${ }^{3}$ Similarly, congenital and acquired dysfibrinogenaemias have been described which may lead to a bleeding or thrombotic state. ${ }^{4-10}$ Further, high levels of normal fibrinogen are an independent risk factor in ischaemic heart disease. ${ }^{11-13}$ Therefore some physicians recommend fibrinogen measurement as part of the profile of thrombo- embolic risk assessment, and this has created an ever increasing demand for fibrinogen assays in the clinical laboratory.

Currently the most widely used technique for determination of fibrinogen concentration is a modification of the assay described by Clauss. ${ }^{14}$ In this method, dilutions of a plasma standard (of known fibrinogen concentration) are clotted with a high concentration of thrombin $(\sim 100 \mathrm{NIH} \mathrm{U} / \mathrm{ml})$, the clotting time being proportional to the fibrinogen concentration; the clotting time of dilute patient's plasma may then be used to read the fibrinogen concentration from the standard curve. The Clauss assay is time consuming, requiring dilution buffer and special thrombin reagent, which on some fully automated coagulation analysers may cause carryover problems in subsequent tests.

In coagulation tests, the change in light scatter or optical density during clot formation shows a progressive increase until a plateau is reached. The height of this response from the baseline is proportional to the fibrinogen concentration. Several modern photo-optical coagulometers exploit this change in optical properties during the prothrombin time reaction to determine a prothrombin time derived (PT derived) fibrinogen concentration, by comparing the response of a test plasma with that of a standard (of known fibrinogen concentration) and extrapolating the fibrinogen level. ${ }^{15-17}$

In this study we undertook a comparative evaluation of Clauss fibrinogen assay versus PT derived fibrinogen determinations on the Sysmex CA-6000 ${ }^{\mathrm{TM}}$. Specific attention was paid to the source of thromboplastin employed in the prothrombin time (that is, to produce the PT derived fibrinogen), and the clinical category of patients from whom samples were obtained-for example, patients with sepsis or a hepatic disorder might have fibrin(ogen) degradation products or an acquired dysfibrinogenaemia that could disturb fibrin polym-

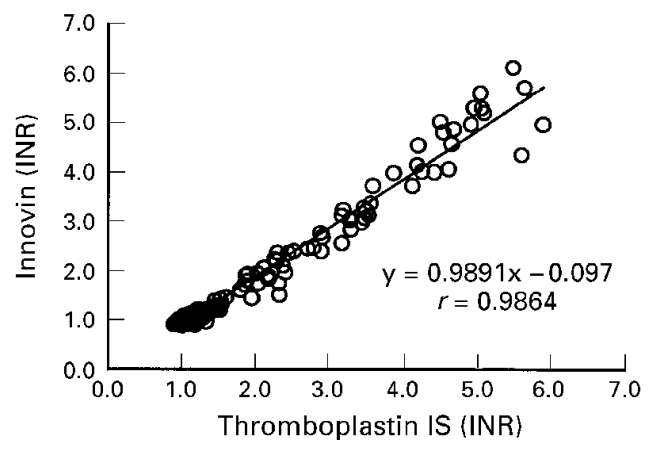

Figure 1 Prothrombin time international normalised ratio (INR) comparability (all samples, $n=247$ ). 

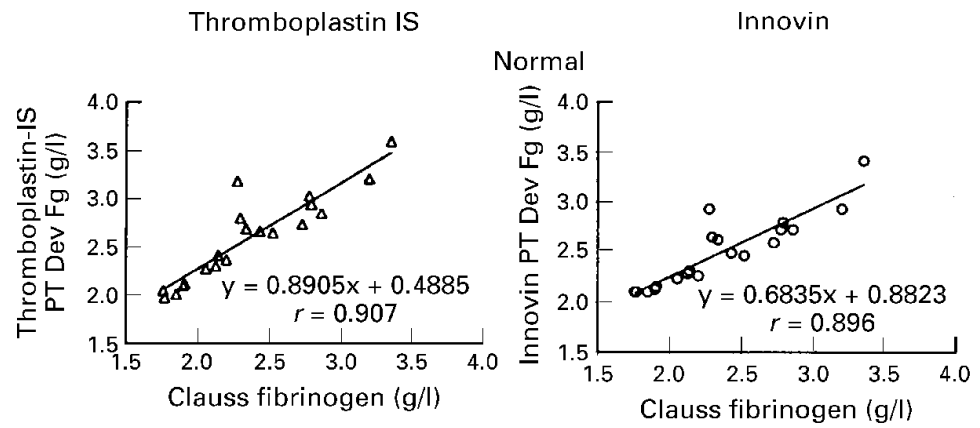

Oral anticoagulant treatment
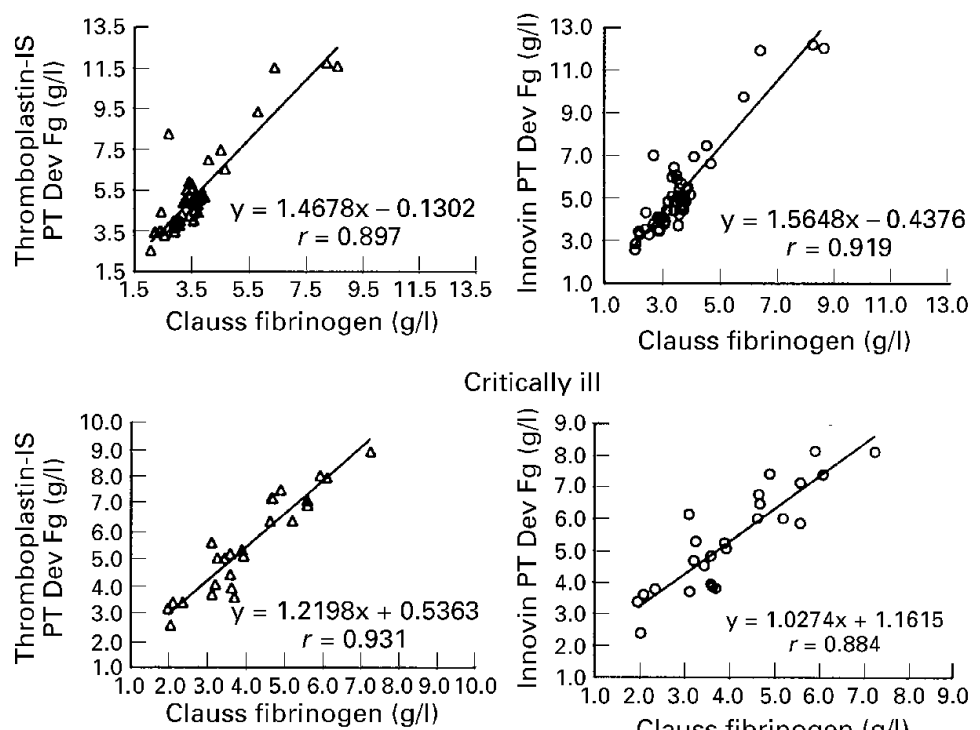

Critically ill

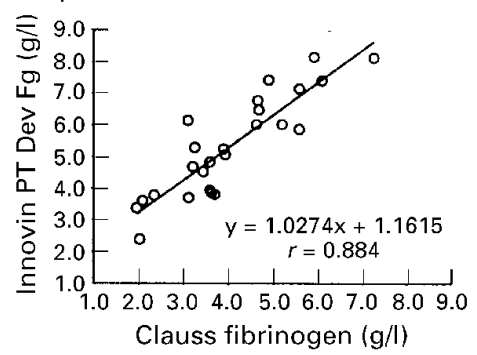

Renal or hepatic dysfunction
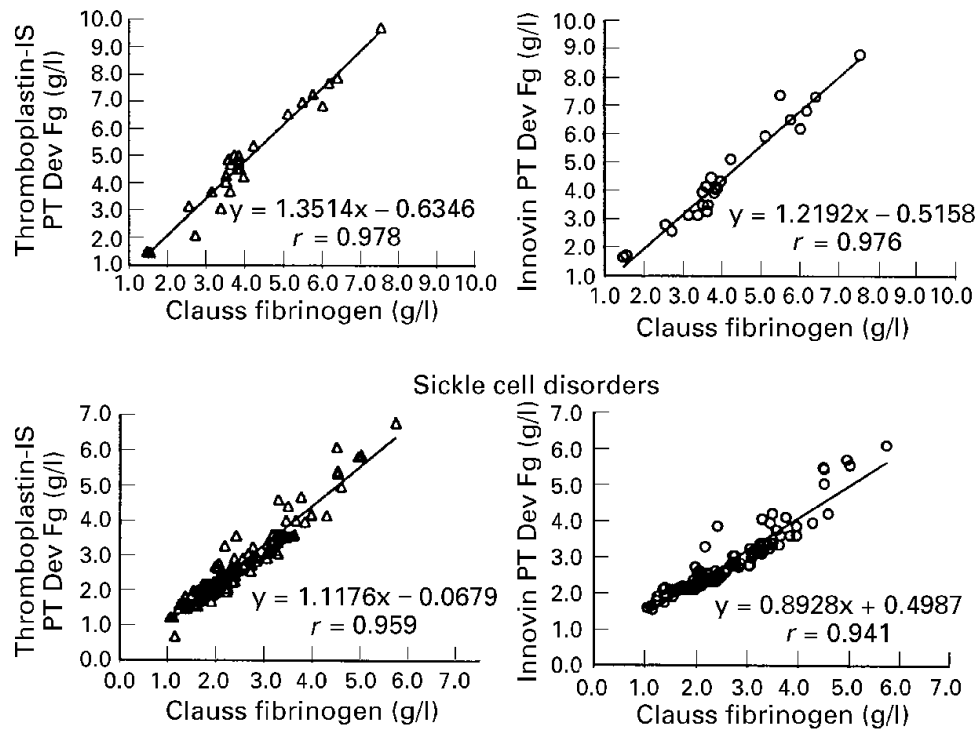

Figure 2 Clinical group dependent correlation between Clauss and prothrombin time derived fibrinogen (PT-dev-Fg).

erisation and affect haemostatic tests based on clot detection. ${ }^{4910}$

\section{Methods}

Samples were obtained from 20 healthy normal subjects, 25 patients with renal or liver dysfunction, 25 critically ill patients, 50 patients receiving oral anticoagulant treatment, and 127 patients with haemoglobinopathies.
The samples from the 50 patients receiving oral anticoagulant treatment were selected to give a range of prothrombin times covering international normalised ratio (INR) values from 1.7 to 5.6. Samples in the haemoglobinopathy group were from 70 patients with sickle cell disorders (these included samples from patients with $\mathrm{HGS} / \beta^{\circ}$ thalassaemia, $\mathrm{HbSD}$, HbSS), 17 with HbSC disease, 15 with $\mathrm{b}^{\circ}$ thalassaemia intermedia, and 25 with $\beta^{\circ}$ thalassaemia major. Twenty of the sickle cell disorders and all of the thalassaemia major patients were on chronic blood transfusion regimens. None of the sickle cell disorders or HbSC patients was in crisis.

Blood was taken by clean venepuncture into $0.105 \mathrm{M}$ trisodium citrate, with a ratio of one part anticoagulant to nine parts whole blood. The samples were centrifuged at $2000 \mathrm{~g}$ for 15 minutes. The upper two thirds of the plasma was removed and subjected to a second centrifugation, also at $2000 \mathrm{~g}$ for 15 minutes. Aliquots of plasma were then stored in polypropylene cryo-tubes $(2.0 \mathrm{ml}$ micro tubes No 72.694.007; Sarstedt, Leicester, UK) for less than three months at $-70^{\circ} \mathrm{C}$ until required for assay, when they were thawed to $37^{\circ} \mathrm{C}$.

Samples were subjected to analysis by the prothrombin time test using two reagents (Thromboplastin IS and Innovin; Dade: Sysmex UK, Milton Keynes, UK) and Clauss fibrinogen assay (Dade). From our previous experience with the above reagents and analyser we anticipated that the prothrombin time reference range clotting times (mean \pm 2 standard deviations) for the different reagents would vary; therefore to facilitate comparison of prothrombin times between reagents, reference ranges were converted to ratios by dividing the upper and lower limits of the range by the range mean.

The prothrombin time ratios (PTR) were calculated using the geometric mean normal for the respective tests. PTR results were converted to INRs with the use of INR calibrated plasmas (AK-Calibrant, Immuno, Sevenoaks, Kent, UK). All analytical procedures were undertaken on a Sysmex CA$6000^{\mathrm{TM}}$ random access coagulation analyser (Sysmex UK) and reagents were used in accordance with their manufacturers' recommendations.

Paired data were analysed using a nonparametric test (Wilcoxon rank sum paired test) and probability (p) $<0.05$ was considered significant. Comparison of results between

Table 1 Reference ranges $(n=20)$

\begin{tabular}{|c|c|c|}
\hline Test & $\begin{array}{l}\text { Geometric } \\
\text { mean }\end{array}$ & $\begin{array}{l}95 \% \text { Confidence } \\
\text { intervals }\end{array}$ \\
\hline PT (s), Thromboplastin IS & 11.7 & 10.6 to 12.9 \\
\hline $\begin{array}{l}\text { PT (ratio), Thromboplastin } \\
\text { IS }\end{array}$ & 1.00 & 0.91 to 1.10 \\
\hline $\begin{array}{l}\text { PT-dev-Fg, Thromboplastin } \\
\text { IS }(\mathrm{g} / \mathrm{l})\end{array}$ & 2.56 & 1.81 to 3.62 \\
\hline PT (s), Innovin & 8.1 & 7.0 to 9.3 \\
\hline PT (ratio), Innovin & 1.00 & 0.87 to 1.15 \\
\hline PT-dev-Fg, Innovin (g/1) & 2.47 & 1.89 to 3.25 \\
\hline Clauss fibrinogen $(\mathrm{g} / \mathrm{l})$ & 2.32 & 1.54 to 3.49 \\
\hline
\end{tabular}

PT, prothrombin time; PT-dev-Fg, prothrombin time derived fibrinogen. 
Table 2 International normalised ratios (INR) and fibrinogen levels on all samples $(n=247)$

\begin{tabular}{|c|c|c|c|c|c|}
\hline & \multicolumn{2}{|c|}{ Thromboplastin IS } & \multicolumn{2}{|c|}{ Innovin } & \multirow{2}{*}{$\begin{array}{l}\text { Clauss fibrinogen } \\
(\mathrm{g} / \mathrm{l})\end{array}$} \\
\hline & INR & $P T-d e v-F g(g / l)$ & INR & $P T-\operatorname{dev}-F g(g / l)$ & \\
\hline Mean & 1.68 & 3.69 & 1.57 & 3.64 & 3.02 \\
\hline SD & 1.10 & 1.91 & 1.10 & 1.81 & 1.27 \\
\hline Minimum & 0.88 & 0.68 & 0.87 & 1.54 & 1.05 \\
\hline Maximum & 5.87 & 11.73 & 6.11 & 12.18 & 8.60 \\
\hline
\end{tabular}

INR were calculated using INR calibrated plasmas. PT-dev-Fg, prothrombin time derived fibrinogen.

Table 3 Variation between fibroginogen determinations

\begin{tabular}{llll}
\hline \multirow{2}{*}{ Sample groups } & \multicolumn{2}{l}{ PT-dev-Fg v Clauss } & PT-dev-Fg \\
\cline { 2 - 3 } & TP-IS & Innovin & TP-IS v Innovin \\
\hline Normal samples & $\mathrm{p}=0.0001$ & $\mathrm{p}=0.009$ & $\mathrm{p}=0.004$ \\
Renal or liver dysfunction & $\mathrm{p}=0.0004$ & $\mathrm{p}=0.001$ & $\mathrm{p}=0.0004$ \\
Critical illness & $\mathrm{p}<0.0001$ & $\mathrm{p}<0.0001$ & $\mathrm{NS}$ \\
Oral anticoagulants & $\mathrm{p}<0.0001$ & $\mathrm{p}<0.0001$ & $\mathrm{NS}$ \\
Haemoglobinopathy & $\mathrm{p}<0.0001$ & $\mathrm{p}<0.0001$ & $\mathrm{NS}$ \\
All samples & $\mathrm{p}<0.0001$ & $\mathrm{p}<0.0001$ & $\mathrm{NS}$
\end{tabular}

PT, prothrombin time; PT-dev-Fg, prothrombin time derived fibrinogen; TP-IS, Thromboplastin IS. jects using Thromboplastin IS and Innovin. When local analyser specific ISI (international sensitivity index) assignment had been undertaken (Thromboplastin IS ISI = 1.15; Innovin ISI $=0.87)$, INR results from the two reagents correlated well $(r=0.99)$ with a slope close to 1.0 and an intercept close to zero (fig 1). However, there was a statistically significant difference between reagents $(p<0.0001)$.

Reference ranges for PT derived fibrinogen and Clauss fibrinogen determinations were established by testing plasma from 20 apparently healthy normal subjects. The ranges were found to be similar for each of these procedures (table 1). However, the relation between PT derived fibrinogen and Clauss fibrinogen was seen to vary markedly between patient groups and was also dependent on the reagent used (fig 2). For a more complete picture of the patients' samples analysed, ranges of PT derived fibrinogen and Clauss fibrinogen are presented in table 2 .

When comparing PT derived fibrinogen results obtained using thromboplastins of different source (Innovin versus Thromboplastin IS), a good correlation was observed in each sample group ( $r=0.97$ to 0.99 , slope $=0.76$ to 1.03 , intercept $=0.13$ to 0.58 ). However, the relation between Clauss fibrinogen and PT derived fibrinogen was seen to be dependent on the sample group and the source of the thromboplastin used for the latter procedure, that is, either Innovin $(r=0.88$ to 0.97 , slope $=$ 0.68 to 1.56 , intercept $=0.25$ to 1.16$)$ or Thromboplastin IS $(r=0.89$ to 0.97 , slope $=$ 0.89 to 1.40 , intercept $=0.06$ to 0.63 ) $($ fig 2$)$.
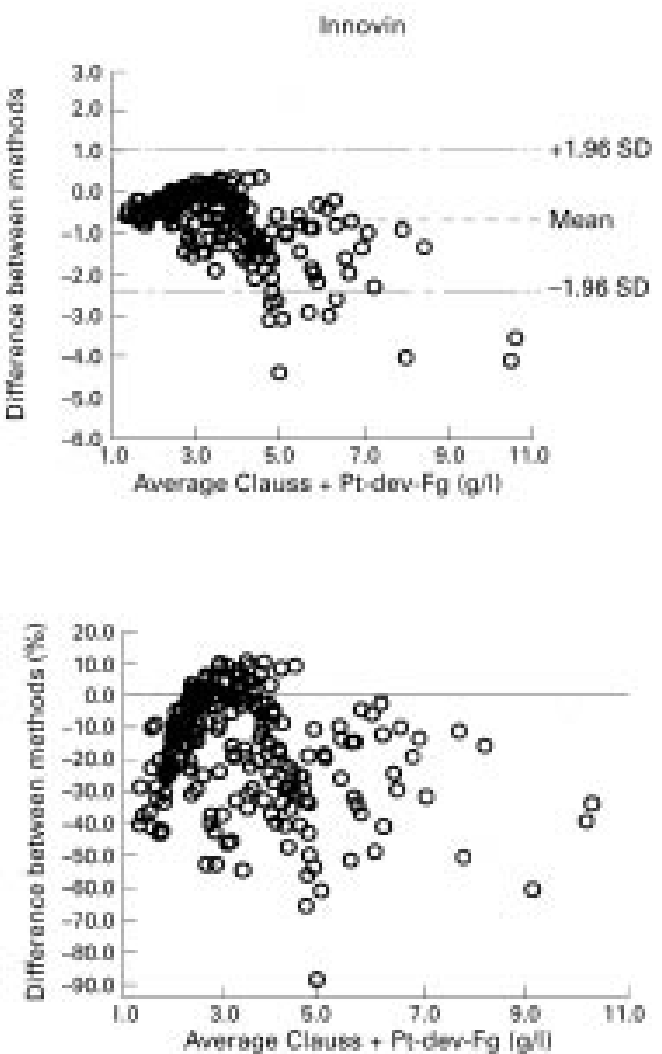

Figure 3 Comparison of Clauss and prothrombin time derived (PT-dev-Fg) methods (after Altman and Bland ${ }^{18}$ ). 
Thromboplastin IS
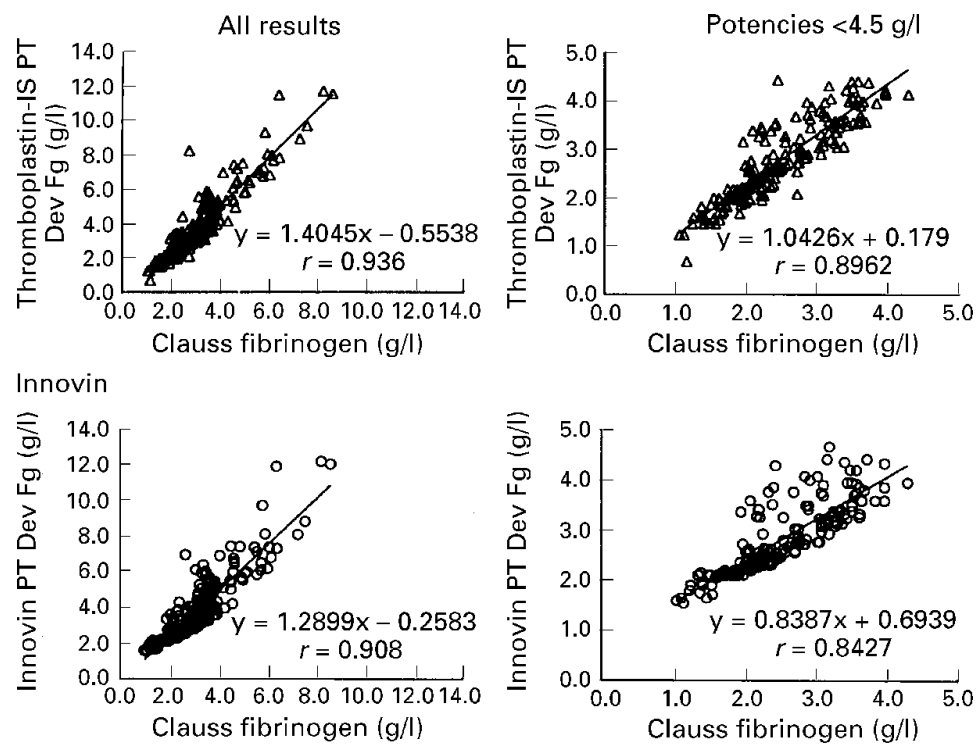

Thromboplastin IS $v$ Innovin

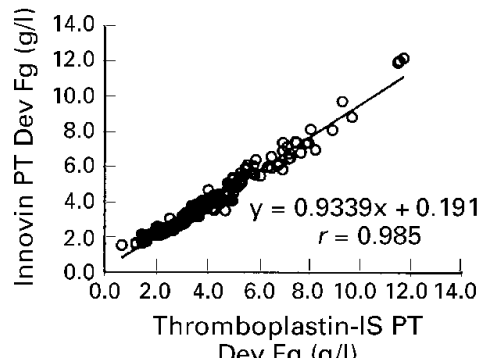

$\operatorname{Dev} \mathrm{Fg}(\mathrm{g} / \mathrm{l}\rangle$
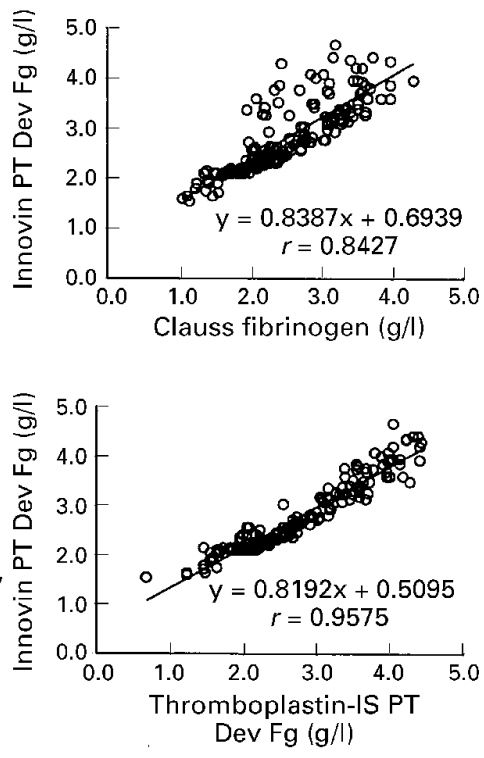

Figure 4 Comparison of Clauss and prothrombin time derived (PT-dev-Fg) methods (all sample groups). are inherently dynamic. Fibrinogen levels in these patients may be increased in response to inflammation or reduced owing to consumption, making accurate determination essential as they provide a means of monitoring the extent of coagulation activation and the clinical course of the disease. Additionally, patients with sickle cell disease or a thalassaemic syndrome suffer chronic mild haemolysis and subsequent exposure to free circulating haemoglobin. Free haemoglobin prolongs the conversion of fibrinogen to fibrin by thrombin up to twofold when present in concentrations as low as $0.48 \mathrm{mM} .{ }^{20}$ As haemolysed erythrocytes may release up to $2.3 \mathrm{mM}$ free haemoglobin, monitoring of fibrinogen levels in these patients may provide an indication of the extent of any low grade subclinical haemolysis occurring.

The PT derived fibrinogen technique uses the difference in light scattering before and after clot formation compared with the readings taken using a standard (of known fibrinogen concentration) and extrapolates the fibrinogen concentration. ${ }^{15}$ PT derived fibrinogen (as its name implies) is not therefore a direct measurement of fibrinogen and it has previously been shown to overestimate fibrinogen concentrations. ${ }^{17}$

This study showed that results from PT derived fibrinogen differed from those obtained by the Clauss technique. The precise relation between the two tests was reliant upon the source of thromboplastin used within the PT derived fibrinogen procedure and the patients' clinical status. As the discrepancy between results obtained by the two techniques could be affected by the patient's clinical state (and a patient's clinical condition can on occasions change rapidly), it would be unsafe to use the PT derived fibrinogen in the clinical environment where the Clauss technique is well established. of thromboplastin $(p<0.001)$. Furthermore, when all sample groups were combined the relation was shown to depend not just on the reagent used (fig 3) but also on the range of fibrinogen levels analysed (for example, $<4.5 \mathrm{~g} / \mathrm{l})$ as shown in fig 4 .

There was no apparent relation between PT derived fibrinogen (using either Thromboplastin IS $(r=0.35)$ or Innovin $(r=0.42)$ ) or Clauss fibrinogen $(r=0.14)$ and the INR value. Similarly when the difference between PT derived fibrinogen (using either Thromboplastin IS $(r=0.48)$ or Innovin $(r=0.48))$ and Clauss fibrinogen was calculated and compared with the INR, no clear relation was shown. Furthermore, correlation between PT derived fibrinogen minus Clauss fibrinogen and Clauss fibrinogen was extremely poor, with considerable variation around the line of regression (using either Thromboplastin IS ( $r$ $=0.60)$ or Innovin $(r=0.44)$ ).

\section{Discussion}

Fibrinogen, an acute phase reactant, is increased in response to both acute and chronic inflammatory processes. ${ }^{19}$ This characteristic is particularly significant in the groups of patients we studied, in whom fibrinogen levels

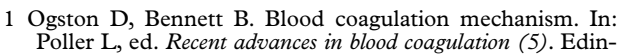
burgh: Churchill Livingstone 1991:1-15.

2 Peerschke EI. The platelet fibrinogen receptor. Semin Hematol 1985;22:241-59.

3 Al-Mondhiry H, Ehmann WC. Congenital afibrinogenemia. Am f Hematol 1994;46:343-7.

4 Dymock IW, Tucker JS, Woolf IL, et al. Coagulation studies as a prognostic index in acute liver failure. Br $\mathcal{F}$ Haematol 1975;29:385-95.

5 Siegal T, Seligsohn U, Aghai E, et al. Clinical and laboratory aspects of disseminated intravascular coagulation (DIC): a study of 118 cases. Thromb Haemost 1978;39:122-34. Biol Res 1981;72:205-20.

7 Bithell TC. Hereditary dysfibrinogenemia. Clin Chem 1985; 31:509-16.

8 Haverkate F, Samama M. Familial dysfibrinogenemia and thrombophilia. Report on a study of the SSC subcommitthrombophilia. Report on a study of the SSC subcom
tee on fibrinogen. Thromb Haemost 1995;73:151-61.

9 Green G, Thomson JM, Dymock IW, et al. Abnormal fibrin preen $G$, Thomson JM, Dymock IW, et al. Abnormal fibrin
polymerisation in liver disease. Br f Haematol 1976;34: 427-39.

10 Lane DA, Scully MF, Thomas DP, et al. Acquired dysfibrinogenaemia in acute and chronic liver disease. $\mathrm{Br} \mathcal{F}$ Haematol 1977;35:301-8.

11 Banerjee AK, Pearson J, Gilliland EL, et al. A six year prospective study of fibrinogen and other risk factors associated with mortality in stable claudicants. Thromb Haemost 1992;68:261-3.

12 Tripodi A, Mannucci PM. Fibrinogen and factor VII as risk factors for ischemic heart disease. Eur $f$ Lab Med factors for ischer
1995;3:262-5.

13 Meade TW. Fibrinogen and cardiovascular disease. $f$ Clin Pathol 1997;50:13-15.
6 Ménaché D. Congenital abnormal fibrinogens. Prog Clin 
14 Clauss A. Gerinnungsphysiologische schnellmethode zur bestimmung des fibrinogens. Acta Haematol 1957;17:23746.

15 Rossi E, Mondonico P, Lombardi A, et al. Method for determination of functional (clottable) fibrinogen by the new family of ACL coagulometers. Thromb Res 1988;52 453-68.

16 Wallace J, Dahms RA. Study of the PT-derived fibrinogen assay on the Sysmex CA-1000 and CA-5000 automated coagulation analyzers. Sysmex $\mathcal{F}$ Int 1994;4:88-95.

17 Chitolie A, Mackie IJ, Grant D, et al. Inaccuracy of the 'derived' fibrinogen measurement. Blood Coagul Fibrinolysis 1994;5:955-7.

18 Altman DG, Bland JM. Measurement in medicine: the analysis of method comparison studies. Statistician 1983; 32:307-17.

19 Kushner I. The acute phase response: an overview. Methods Enzymol 1988;163:373-6.

20 Brezniak DV, Moon DG, Beaver JA, et al. Haemoglobin inhibition of fibrin polymerization and clotting. Blood Coagul Fibrinolysis 1994;5:139-43. 